\section{$\underset{\substack{\text { hommes } \\ \text { \& migrations }}}{ }$}

\section{Hommes \& migrations}

Revue française de référence sur les dynamiques

migratoires

1303 | 2013

Diasporas marocaines

\title{
Quand la bande dessinée s'empare de la mémoire
}

Entretien avec Anne-Hélène Hoog

Hélène Bouillon et Marie Poinsot

\section{(e) OpenEdition \\ 1 Journals}

Édition électronique

URL : http://journals.openedition.org/hommesmigrations/2584

DOI : 10.4000/hommesmigrations.2584

ISSN : 2262-3353

Éditeur

Musée national de l'histoire de l'immigration

Édition imprimée

Date de publication : 1 juillet 2013

Pagination : 164-169

ISBN : 978-2-919040-23-0

ISSN : $1142-852 X$

Référence électronique

Hélène Bouillon et Marie Poinsot, "Quand la bande dessinée s'empare de la mémoire », Hommes \& migrations [En ligne], 1303 | 2013, mis en ligne le 23 janvier 2014, consulté le 22 septembre 2020.

URL : http://journals.openedition.org/hommesmigrations/2584; DOI : https://doi.org/10.4000/ hommesmigrations.2584 


\section{ENTRETIEN}

\section{QUAND LA BANDE DESSINÉE S'EMPARE DE LA MÉMOIRE}

ENTRETIEN AVEC ANNE-HÉLÈNE HOOG, réalisé par Hélène Bouillon et Marie Poinsot

A nne-Hélène Hoog est conservatrice au Musée d'art et d'histoire du judaïsme. Après des études d'histoire à Grenoble et à l'EHESS, de langues et culture juives à l'Inalco, elle a travaillé plusieurs années en Allemagne pour des institutions publiques conservant des collections juives. Elle rejoint en janvier 1998 le Musée d'art et d'histoire du judaïsme, où elle a organisé depuis plusieurs expositions. Revenant dans cet entretien sur sa première exposition de bande dessinée, De Superman au Chat du Rabbin, Anne Hélène Hoog défend la place de l'art séquentiel au musée et évoque ces artistes qui s'expriment et témoignent à coups de crayon.

Hommes \& Migrations : Comment se sont constituées les collections du Musée d'art et d'histoire du judaïsme?

Anne-Hélène Hoog : Le Mahj a repris les collections de diverses institutions. Tout d'abord, il a hérité de celles du Musée d'art juif qui avait été fondé en 1948 et qui avait recueilli les dons de membres de la communauté juive française dans le souci de préserver ce qui restait non seulement des artistes juifs disparus pendant la guerre, mais plus généralement du judaïsme. Outre la constitution d'une collection en propre, le musée a également bénéficié de dépôts de la part d'institutions communautaires mais aussi du Musée national du Moyen Âge, du Musée national d'art moderne, du Musée historique lorrain et du musée Carnavalet.
$H \& M$ : Quand et comment vous êtes-vous intéressée au neuvième art?

A.-H. H. : Je connaissais la bande dessinée comme lectrice, sans en être une fan. L'œuvre d'Hugo Pratt surtout m’a impressionnée. Mais, plus généralement, je dirais que l'alliance de l'art et de la littérature m'est toujours apparue comme naturelle. Le texte et l'image se complètent, se révèlent et s'épaulent l'un l'autre. L'image parle à celui qui la regarde. Le message délivré donne un horizon intellectuel, spirituel, historique, une vision du monde ou d'un aspect particulier.

$H \& M$ : Quelle est la genèse de l'exposition De Superman au Chat du Rabbin?

A.-H. H. : L'exposition De Superman au Chat $d u$ Rabbin a été précédée d'une période de gestation d'environ cinq ans. Depuis l'exposition de 2002 Le Juif errant, un témoin du temps, je me disais que nous utilisions des images, mais que nous ne les interprétions pas suffisamment comme témoignages ou récits mémoriels et qu'il nous fallait reconnaître et analyser leur vocabulaire pour en comprendre le sens et comprendre comment elles sont "encodées" par l'artiste puis décodées par celui qui les regarde. C'est pourquoi je parle de "codes visuels" de la bande dessinée. Évidemment, la narration par l'image séquentielle pourrait se passer de tout texte. Seulement, il peut être important d'avoir quelque chose en plus. La bande dessinée et surtout le roman graphique présentent un grand intérêt parce que le texte est 
dans le dessin et qu'il en fait complètement partie. En outre, il y a un style, une écriture et une esthétique du lettrage, un travail de composition de la page tout à fait parlant. Ce sont autant de témoignages de la manière dont un artiste essaye de nous donner une vision de ce que lui-même a perçu auparavant. II est un passeur d'images, un passeur de sens, et indiscutablement, un passeur d'histoire(s).

Étant donné que les auteurs de bandes dessinées travaillent souvent à partir de documents préexistants, leur démarche nous offre l'opportunité de nous pencher sur des problématiques telles que l'intégration, l'immigration, le changement culturel ou la transmission de la mémoire. Pour moi, il s'agissait avec la BD d'apporter une certaine vision de l'histoire juive, de l'histoire des parents, de l'histoire des familles à travers des images. Comme des tableaux qu'on donnerait à une génération qui n’a pas connu la difficulté d'être un immigré à New York, de faire tel travail, de vivre dans tel type de famille, etc.
$H \& M$ : La BD a-t-elle une place importante dans l'histoire et la production artistique et culturelle juives?

A.-H. H. : On peut considérer que le roman graphique Un pacte avec Dieu de Will Eisner a été le premier en 1976 publié sur le thème. II a fallu attendre plus longtemps pour Maus d'Art Spiegelman. Mais le travail de la mémoire juive ne peut pas uniquement concerner la Shoah ou ce qu'on veut transmettre du sort particulier des rescapés. Il faut aller plus loin. Dans le corpus de témoignages et de visions proposés aujourd'hui par la BD, il est intéressant de décoder ce qui, dans un monde qui n'est pas juif, peut relever du judaïsme et nous dire quelque chose de fondamental sur les juifs d'aujourd'hui, sur leur vision du monde et du sens de leur existence. Comment certaines idées passent-elles d'une génération à l'autre? Comment la bande dessinée, entre autres, a-t-elle pu, en très peu de signes, résumer des situations de vie, des parcours, des questionnements ? Dans l'histoire juive, la BD a très peu 


\section{ENTRETIEN}

d'importance, ne serait-ce que parce que c'est un médium récent. L'image elle-même n'a longtemps eu de place que dans certains livres de la tradition juive comme la Haggadah de Pâque, les rouleaux d'Esther ou Megillot Esther lus pour la fête de Pourim ou les livres sur les rites dits Minhagim. Ce sont des illus-

La notion de diaspora renvoie moins à l'idée de la mobilité qu'au temps qui passe et qui efface les traces. On retrouve cette fragilité-là évidemment dans les migrations, avec le déracinement ou le changement du temps qu'elles impliquent. trations ayant connu peu d'innovations. Elles sont copiées d'une génération à l'autre, de manière très codifiée puisqu'il s'agit d'extraire l'essentiel d'un rite ou d'une situation et de faire en sorte que les gens en soient marqués. On pense surtout aux enfants puisque l'activité pédagogique est fondamentale dans le judaïsme pour lequel le récit par le texte est au cœur de la transmission. L'image était importante bien qu'elle ne fût pas l'objet d'un soin ou d'un souci de réinterprétation ou d'innovation particuliers.

Avec le projet De Superman au Chat du Rabbin, il a fallu trouver une approche permettant la restitution d'un monde dans lequel les juifs ont été très actifs, sur un mode prolifique, instructif et fructueux. Ce monde fut bien moins celui de la bande dessinée dans le judaïsme que celui des juifs dans la bande dessinée. Pourquoi y sont-ils présents très rapidement et de multiples façons, c'est-à-dire comme artistes, éditeurs, vendeurs ou lecteurs ? Où se trouvent les points de rencontre entre le fait qu'ils soient juifs et leur rôle social ou culturel ? Pourquoi tiennent-ils à parler de leur propre histoire ou à aborder des questions qui leur sont contemporaines ? Je pense à Will Eisner bien sûr, ou à Art Spiegelman. Pour ce dernier, la BD fut une sorte de thérapie graphique. Elle lui a permis de montrer l'affrontement de deux générations, de restaurer le dialogue très douloureux entre un père et son fils. Tout cela sur le fond d'une histoire très trouble, celle des parents, inscrite dans la grande histoire de la Shoah. Le livre Maus de Spiegelman reste un paradigme pour ce qui concerne la mémoire graphique, le roman graphique et le récit sur la Shoah.

Aujourd'hui, Spiegelman n'est plus le seul à faire cette démarche. Nous assistons à une floraison de récits mémoriels à travers la bande dessinée. Les œuvres de Bernice Eisenstein ou Miriam Katin relèvent à la fois de l'œuvre et du témoignage. D'autres cherchent à restituer des périodes avec le roman graphique historique ou historico-fantastique, redevenu un genre, à l'instar de l'histoire elle-même redevenue un genre dans la littérature. Joann Sfar le montre avec Klezmer, Le Chat du Rabbin ou Le Petit Monde du Golem. On voit émerger une nécessité d'évoquer ou de témoigner, qui doit s'appuyer sur des codes spécifiques et suffisamment familiers pour être repérés et déchiffrés par tous ou presque.

$H \& M$ : La dimension diasporique de cette culture juive peut-elle être un élément d'explication de ce mouvement ? Faut-il y voir le besoin de laisser une trace en dépit de ce sentiment de mobilité? A.-H. H. : La notion de diaspora renvoie moins à l'idée de la mobilité qu'au temps qui passe et qui efface les traces. On retrouve cette fragilité-là évidemment dans les migrations, avec le déracinement ou le changement du temps qu'elles impliquent. Le monde d'hier fait place à un monde dont on ne sait pas ce qu'il va advenir. Il n'est pas vraiment le vôtre, vous n'en faites que très peu partie, ou pas pour longtemps. Mais vos enfants y vivront. II y a une volonté de faire en sorte que les générations futures gardent le souvenir de ce qu'ont été celles d'avant. C'est la préoccupation face à cette perte de mémoire collective qui est à l'origine de la collecte d'archives initiée par le Mahj depuis quelques années.

Dans cette démarche, on est bien sûr livré au hasard des rencontres. Les gens sont volontaires 
pour participer à ces collectes et partagent une part de leur propre histoire. Le souci moteur dans la démarche de collecte est de permettre des modes d'interprétation différents. II faut éviter un discours univoque et, au contraire, documenter les parcours et les positionnements individuels. On se rend ainsi compte de la grande complexité des choses, une complexité que l'on devient prêt à son tour à accueillir dans sa réflexion. Par exemple, l'immigration et les modalités d'intégration sont au cœur même des questions contemporaines. Mais elles ne se posaient pas en problèmes de la même façon à l'aube du XIX ${ }^{e}$ siècle, par exemple. En France, on pensait alors que l'éducation permettait à l'être de s'accomplir, et que tout immigré au contact des principes du pays d'accueil s'y reconnaîtrait et adhérerait à cette culture porteuse d'universel. Aujourd'hui, la culture d'expérience de groupe a remplacé l'universalisme. Cette expérience au sein du groupe est aussi diverse que les groupes le sont eux-mêmes dans la société. Dans cette perspective, en tant qu'objet de collection et objet d'étude pour l'historien, la bande dessinée constitue un document, avec toutes les possibilités que celui-ci donne pour répondre aux questions qu'on se pose ou pour en faire émerger de nouvelles.

Hélas pour les chercheurs et pour le public, beaucoup de très belles œuvres ont été vendues planche après planche, ce qui évidemment est un non-sens pour un musée d'histoire tel que le Musée d'art et d'histoire du judaïsme. Car ce n'est pas la planche en soi, cet élément isolé, qui est intéressant ici mais l'élaboration et la cohérence du récit. La planche prend un sens car la ligne graphique répond à une logique intérieure du récit qui ne peut être montrée qu'avec le récit tout entier.

$H \& M$ : Ne serait-il pas intéressant alors de collectionner la planche de BD avec les commentaires de l'auteur, du dessinateur ou du scénariste, qui montreraient le processus de fabrication de cette mémoire et de cet imaginaire traités sous la forme originale de la bande dessinée?

A.-H. H. : La bande dessinée est faite pour être publiée. À l'origine, personne ne prêtait attention aux planches une fois qu'elles avaient été imprimées. Or, quand on a une planche, il est intéressant d'en connaître toutes les étapes avant la phase finale de l'impression. En ce sens, collectionner des planches, pour un musée d'histoire, c'est aussi s'engager à restituer leur genèse. Les croquis et les études préparatoires de ces planches montrent le travail de mise en mémoire. II y a un lien intime entre l'œil, la mémoire et la main qui dessine. L'affrontement des matières, entre le crayon et le papier, oblige l'auteur à composer.

La contrainte exercée mutuellementparlecontenu, les techniques employées et le support adopté est révélatrice : regardez Comme tout artiste, l'auteur de $B D$ veut restituer le plus exactement possible ce qu'il a vu (son expérience visuelle ou sa vision) et ce qu'il veut dire. La BD est aussi un monde où il est possible de sortir des cases pour être au plus près du cœur du propos. les œuvres de Will Eisner.

De ses crayonnés à la version finale, la façon dont il repositionne et recompose images et textes, tout dans son "économie de la planche" dit la volonté de l'auteur d'être le plus complet, intense et exact dans le sens à donner à son œuvre.

Comme tout artiste, l'auteur de BD veut restituer le plus exactement possible ce qu'il a vu (son expérience visuelle ou sa vision) et ce qu'il veut dire. La BD est aussi un monde où il est possible de sortir des cases pour être au plus près du cœur du propos. Par exemple, l'œuvre de Joann Sfar, Le Chat du Rabbin, est organisée en cases, tandis que Klezmer est fait de débordements à toutes les pages.

Dans nombre de romans graphi-ques, on trouve des hors-cadres, des hors-strips. Ils font souvent la force, la dynamique et l'historique du récit. 


\section{ENTRETIEN}

H\&M : Dans la préparation d'une exposition sur la bande dessinée, qu'est-ce qui fait qu'on décide, pour un artiste, de n'utiliser qu'une planche, et plutôt quatre pour un autre ? Ces choix sont-ils faits avec les artistes?

A.-H. H. : Le rôle d'un commissaire d'exposition est d'appuyer ses choix sur la vision qu'il veut défendre. Ces choix sont toujours arbitraires. On n'est pas commissaire d'exposition sans, à un moment ou à un autre, s'impliquer d'une façon plus personnelle ; plus particulièrement lorsqu'on est en même temps commissaire d'exposition et responsable d'une collection. On n'est pas non plus seul face à toutes

Les images ne sont pas seulement de la monstration mais des guides et des porteurs, comme les pierres d'un gué pour traverser l'exposition et son propos.

Et vous ne donnez pas seulement des instruments et des outils de compréhension, vous ouvrez aussi des univers

qu'éventuellement vousmême ne soupçonnez pas.

de la monstration mais des guides et des porteurs, comme les pierres d'un gué pour traverser l'exposition et son propos. Et vous ne donnez pas seulement des instruments et des outils de compréhension, vous ouvrez aussi des univers qu'éventuellement vous-même ne soupçonnez pas. Mais le résultat n'est jamais garanti.

Pour une exposition comme De Superman au Chat du Rabbin je n'ai pas recherché l'exhaustivité mais j'ai choisi de ne proposer qu'un moment clé de l'œuvre et, à travers celui-ci, d'aborder différents thèmes. Par moments, je ne montrais que les étapes intermédiaires ou je privilégiais l'aspect esthétique. Certaines planches sont d'une telle beauté que vous ne pouvez renoncer à les mon- trer sous prétexte qu'elles ne disent pas ce que vous recherchez. II faut trouver un équilibre entre l'esthétique et le sens.

H\&M : Une exposition comme De Superman au Chat du Rabbin a-t-elle changé le rapport du public à votre musée?

A.-H. H. : L'exposition a permis de toucher un public nouveau qui, sans être fidèle, n'est plus étranger au lieu. Le rôle d'un musée est de parler à tous et en ce sens, il n'y a pas de public cible car nous voulons tous les publics pour toutes les expositions. Je ne conçois pas de faire une exposition pour un groupe d'âge ou un groupe socioculturel. Le challenge d'une exposition comme médiation, c'est de parler à tout le monde et de faire sens pour le plus de visiteurs possible.

En 2014, le Musée d'art et d'histoire du judaïsme mettra à I'honneur le dessinateur Gotlib. Gotlib et son ami et agent Michel Lieuré ont proposé au Mahj de faire une exposition après avoir vu De Superman au Chat du Rabbin. Plus d'une centaine de planches originales de l'artiste vont y être présentées ainsi que de nombreuses archives photographiques, écrites et audiovisuelles. Ce n'est pas à proprement parler une rétrospective de son œuvre, mais une évocation de son parcours d'homme et d'artiste. Pour un artiste, cela implique d'être touché personnellement, de dévoiler des détails de sa vie, de son rapport aux autres, à ses parents, mais aussi l'histoire de ses origines.

Gotlib a été ce galopin qui a grandi entre les rues Ramey et Ferdinand-Flocon du XVIII arrondissement, qui a été un enfant caché pendant la guerre et qui a fait sa bar mitzvah après la guerre dans un home pour enfants tenus par des juifs hongrois. II l'a dit, le fait d'être juif est important pour lui, mais il ne pense pas que cela doive l'être pour les autres. À cet égard, il a un raisonnement qui est très fréquent en diaspora mais aussi en Israël : on est juif, et on est libre de décider de ce 
qu'on veut faire et de ce qu'on veut être. La pensée et le dessin de Gotlib attestent une histoire juive mais aussi très universelle. Je veux dire qu'on n'a pas besoin d'être juif pour avoir ce type de regard sur le monde, mais les juifs l'ont plus profondément et plus souvent que d'autres parce que leur histoire ne leur a pas laissé d'alternative. Comme vous le remarquiez, une exposition Gotlib pourrait avoir lieu à Angoulême, mais au Mahj, elle s'inscrit dans une approche différente. Nous ne voulons pas faire une rétrospective, même si l'artiste nous a fait l'honneur de nous prêter plus d'une centaine d'originaux inédits. Un article récent disait "enfin quelque chose de drôle au musée". Je ne suis pas sûre de vouloir faire quelque chose de drôle. Simplement, l'enfant Marcel Gotlieb a eu un parcours difficile (qu'il a remarquablement supporté), puis Gotlib a trouvé l'art et la manière d'en faire quelque chose de très drôle. Mon propos, biographie mise à part, est d'interroger son humour à la fois potache et amer, très anglo-saxon, souvent impitoyable mais sans méchanceté, nourri par l'autodérision. Une posture que l'on retrouve chez nombre des auteurs de comics juifs d'outreAtlantique dans les années 1960-1970 et jusqu'à présent pour certains d'entre eux. Elle consiste à placer sa propre personne sous une loupe comme l'objet misérable et pathétique de son propre examen. Nul recours face à une telle situation. C'est le comique du tragique, tellement dérisoire qu'on ne peut plus qu'en rire. II y a une espèce d'accablement et d'impuissance du personnage qui fait ce qu'il peut pour s'extraire et se moquer de lui-même. Le dessin, le choix des textes ou même le lettrage permettent une expression sensible. Gotlib, rappelons-le, est un grand lettreur. Pour lui, la lettre est une esthétique en soi. Cela va au-delà de la calligraphie. II s'agit d'une occupation de l'espace, la lettre est un signe, mais plus encore, elle est un son. Dans ses $B D$, le texte et le dessin font son. Voilà, par exemple, un de mes critères pour décider si un auteur m'intéresse.

J'ai toujours pensé que l'exposition De Superman au Chat du Rabbin ne faisait qu'initier une façon de faire parler la bande dessinée, de la prendre comme témoignage. D'autres histoires pourraient être racontées de la sorte : l'histoire de l'immigration, l'histoire des Tziganes ou encore celle des femmes. En cela, par exemple, le travail d'Yvan Alagbé, comme artiste et comme éditeur, est exemplaire. II fait travailler des artistes et lui-même dessine et écrit des scénarios qui parlent des paradoxes de l'immigré, de son parcours, de son malaise. La qualité artistique des œuvres qu'il choisit d'éditer est remarquable. On peut ainsi expliquer les codes visuels liés à la perception de ces groupes et à leurs expériences individuelles, mais également leur réinvestissement ou leur modification par de nouveaux langages. Ici, du coup, je ne m'intéresse pas à la répétition, je m'intéresse aux nouveaux investissements du sens. 